\title{
Dynamical Formation of Spatially Localized Arrays of Aligned Nanowires in Plastic Films with Magnetic Anisotropy
}

Despina Fragouli, ${ }^{\dagger, *}$ Raffaella Buonsanti, ${ }^{ \pm, s}$ Giovanni Bertoni, ${ }^{\perp}$ Claudio Sangregorio, Claudia Innocenti, Andrea Falqui, ${ }^{\perp}$ Dante Gatteschi," Pantaleo Davide Cozzoli, ${ }^{\neq, s}$ Athanassia Athanassiou, ${ }^{t, \S}$ and Roberto Cingolani ${ }^{\mathrm{t}, \perp}$

${ }^{\dagger}$ Center for Bio-Molecular Nanotechnologies (CBN) of IIT@NNL-UniLe-Lecce, via Barsanti c/o STAMMS, Arnesano 73010 Lecce, Italy, ”Scuola Superiore ISUFI, Università del Salento, Distretto Tecnologico ISUFI, ${ }^{\circledR}$ National Nanotechnology Laboratory of CNR-INFM, Unità di Ricerca IIT, via per Arnesano km 5, 73100 Lecce, Italy, ${ }^{\perp}$ Fondazione Istituto Italiano di Tecnologia (IIT), via Morego 30, I-16163 Genova, Italy, and IINSTM Research Unit-Dipartimento di Chimica, Università di Firenze, via della Lastruccia 3 , 50019 Sesto F.no, Firenze, Italy

I norganic nanoparticles (NPs) are excellent candidates for building blocks in nanocomposite materials with engineered physicochemical properties. In particular, nanocomposites of polymers and magnetic NPs can pave the way to novel plastic devices for data storage, ${ }^{1}$ sensing, ${ }^{2}$ actuation, ${ }^{3}$ molecular separation, ${ }^{4}$ and electromagnetic wave absorption. ${ }^{5}$ For enhanced performances such systems require a high density of oriented anisotropic magnetic nanostructures, like nanowires (NWs), in polymer films. One-dimensional (1D) magnetic NWs can be produced by the assembly of isotropic magnetic NPs. ${ }^{6}$ This assembly for NPs dispersed in solution can be done under external magnetic field (MF) during solvent evaporation..$^{7-11}$ This is an attractive technique for the fabrication of NWs, due to its simplicity and effectiveness, compared to other methods such as molecular linker induced assembly ${ }^{12,13}$ or templating techniques. ${ }^{14,15}$ The application of an external magnetic field was formerly used for the orientation of micrometersized particles in polymer matrices for the creation and alignment of wires. ${ }^{16}$ Nevertheless, the use of smaller particles in the nanoscale resulted so far in oriented supra-aggregates. ${ }^{3,17}$ Here we demonstrate by using this method how the NP assembly into magnetic NWs can be controlled in a polymer matrix to achieve reproducible arrays of NWs specifically positioned throughout the volume of polymer films. The structural evolution, ordering, and positioning of www.acsnano.org
ABSTRACT We present a simple technique for magnetic-field-induced formation, assembling, and positioning of magnetic nanowires in a polymer film. Starting from a polymer/iron oxide nanoparticle casted solution that is allowed to dry along with the application of a weak magnetic field, nanocomposite films incorporating aligned nanocrystal-built nanowire arrays are obtained. The control of the dimensions of the nanowires and of their localization across the polymer matrix is achieved by varying the duration of the applied magnetic field, in combination with the evaporation dynamics. These multifunctional anisotropic free-standing nanocomposite films, which demonstrate high magnetic anisotropy, can be used in a wide field of technological applications, ranging from sensors to microfluidics and magnetic devices.

KEYWORDS: colloidal nanoparticles · polymers · magnetic field $\cdot$ directed assembly · nanowires - magnetic nanocomposites

the NWs within the films are controlled by the application time of an external MF during solvent evaporation from a polymer/ magnetic NP solution and by the viscosity gradient across the formed film.

In particular, we present the MF-driven fabrication of plastic films, thick enough to be free-standing, that contain 1D arrays of aligned magnetic NP-built NWs with magnetically anisotropic behavior. We have exploited the magnetically induced assembly of monodisperse hydrophophic-capped spherical NPs of iron oxide in the spineltype cubic structure (henceforth named as $\gamma-\mathrm{Fe}_{2} \mathrm{O}_{3}$ ) with a mean diameter of $10 \mathrm{~nm}$ (see Supporting Information Figure S1), in a polymer matrix, which under suitable experimental conditions results in the controlled formation of aligned NWs selectively distributed across the polymer film thickness. Specifically, the presented procedure offers the possibility to create, align, and
*Address correspondence to despina.fragouli@unile.it, despina.fragouli@iit.it.

Received for review November 11, 2009 and accepted March 18, 2010.

Published online March 31, 2010. $10.1021 / \mathrm{nn} 901597 a$

(c) 2010 American Chemical Society 


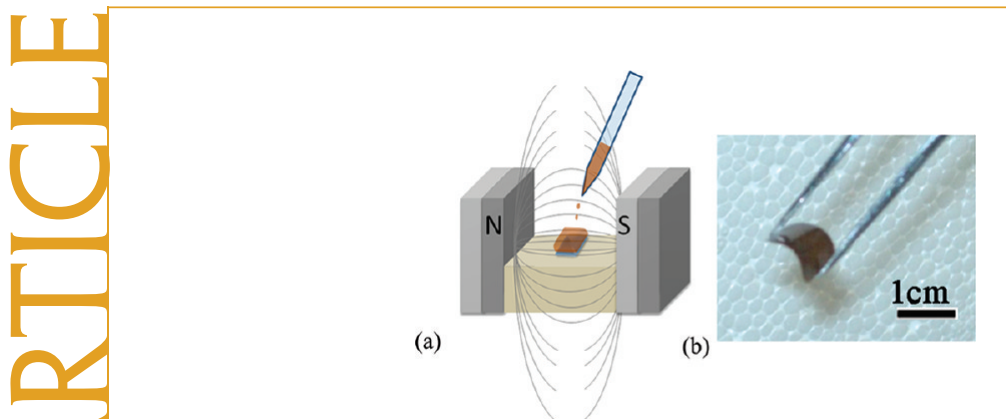

Figure 1. (a) Sketch of the fabrication method; (b) photograph of a freestanding nanocomposite film with an average thickness of $100 \mu \mathrm{m}$

locate NWs in plastic matrices. This is a promising route toward the realization of magnetically anisotropic freestanding functional films, which can directly be peeled from their substrate without altering the aligned NP structures therein.

\section{RESULTS AND DISCUSSION}

The nanocomposite films are formed during solvent drying from casted solutions of different NP/polymer combinations in chloroform, under a weak MF (160 $\mathrm{mT}$ ) as presented in the Supporting Information (see Figure S2). Herein we present analytically the formation of NWs in nanocomposite films composed by $\gamma-\mathrm{Fe}_{2} \mathrm{O}_{3}$ /poly(ethylmethacrylate-co-methylacrylate) (PEMMA) (Figure 1). The morphology of the films is highly reproducible and depends mainly on the NP concentration and on the time of exposure the MF. In general NP concentration lower than $0.7 \%$ in weight results in short elongated structures with random alignment, whereas concentrations between $2 \%$ and $5 \%$ normally permit the formation of ordered arrays of 1D NWs (see Supporting Information Figure S3).

As aforementioned, the duration of the MF application time during the solvent evaporation from the casted film also plays a crucial role in the size and volumetric distribution of the wires in the matrix. The $1 \mathrm{wt}$ $\% \gamma-\mathrm{Fe}_{2} \mathrm{O}_{3} /$ PEMMA nanocomposite films formed upon evaporation without MF application contain inhomogeneous islandlike structures of NPs without a defined shape as shown at the optical microscopy and BF-TEM images in Figure 2a,b. By applying a MF throughout the deposition and solvent evaporation stages, the film morphology changes markedly. The magnetic force modifies the structural shape and causes the formation of aligned NWs with a mean diameter of $c a .80 \mathrm{~nm}$ and length of $15 \mu \mathrm{m}$, uniformly dispersed in the whole polymer matrix (Figure $2 \mathrm{e}, \mathrm{f}$ ) with a density of $1.2 \%$ in the film volume. In any case, in the absence of the polymer the formed NWs look shorter and very sparse demonstrating the crucial role of the matrix in the presented procedure (see Supporting Information Figure S4).

To facilitate the NW formation in the polymer matrix it is important that the process starts with the organization of the NPs into bigger clusters. The latter exhibit higher magnetic moments than the single NPs, resulting in an increased response to the MF that leads eventually to chain formation. ${ }^{18}$ In the present case the long alkyl-chain capping molecules (namely oleic acid, oleylamine, and hexadecane-1,2-diol) that surround the NPs make the contact distance between them larger than what is mentioned in the literature as the distance needed for the clusters to be formed $(<1.4 \mathrm{~nm}$ ) due to van der Waals (VdWs) interactions among them. ${ }^{18} \mathrm{How}$ ever, since we use an acrylic polymer whose methyl carboxylate groups do not interact with the capping molecules, mutual hydrophobic interactions among the alkyl chains are expected to prevail, inducing local NP
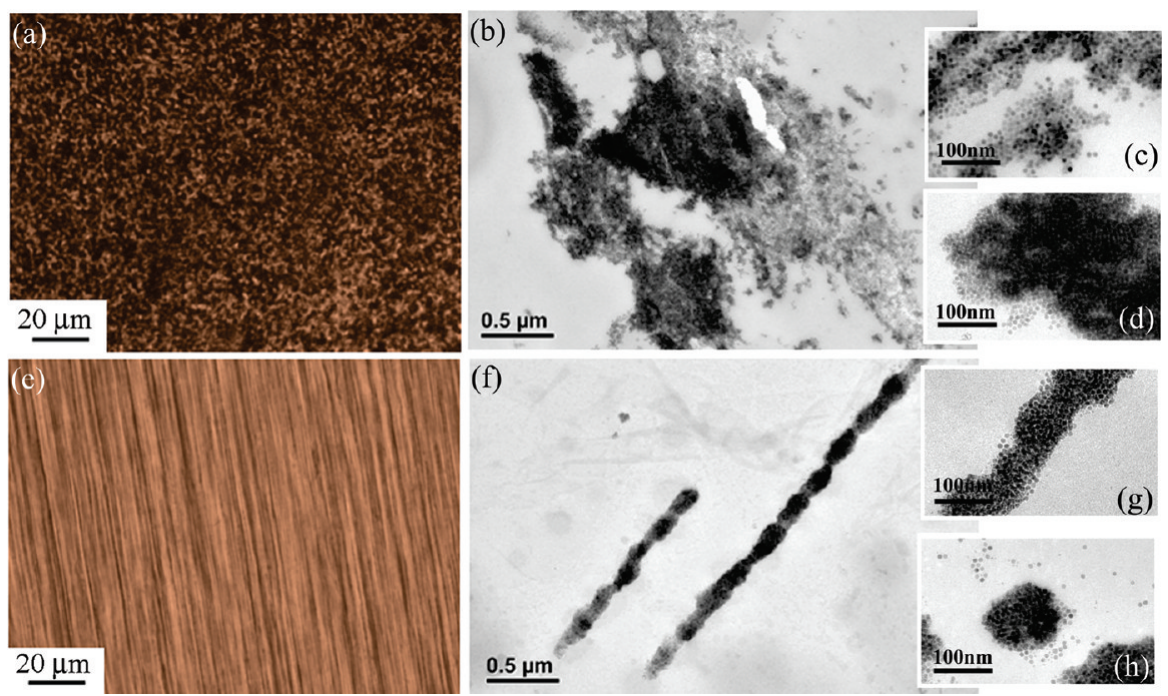

Figure 2. (a, b) Optical microscopy and BF-TEM images, respectively, of a film formed without MF application. The two insets of panel $b$ correspond to higher magnification TEM images: (c) image taken parallel to the film plane; (d) image from a $100 \mathrm{~nm}$ thick cross section, showing the aggregates on a perpendicular plane. (e, f) Optical and BF-TEM images of the film formed upon overnight evaporation under a weak MF; (g) image taken parallel to the NWs plane; (h) image from a $100 \mathrm{~nm}$ thick cross section, showing the NWs perpendicular to their axis. 

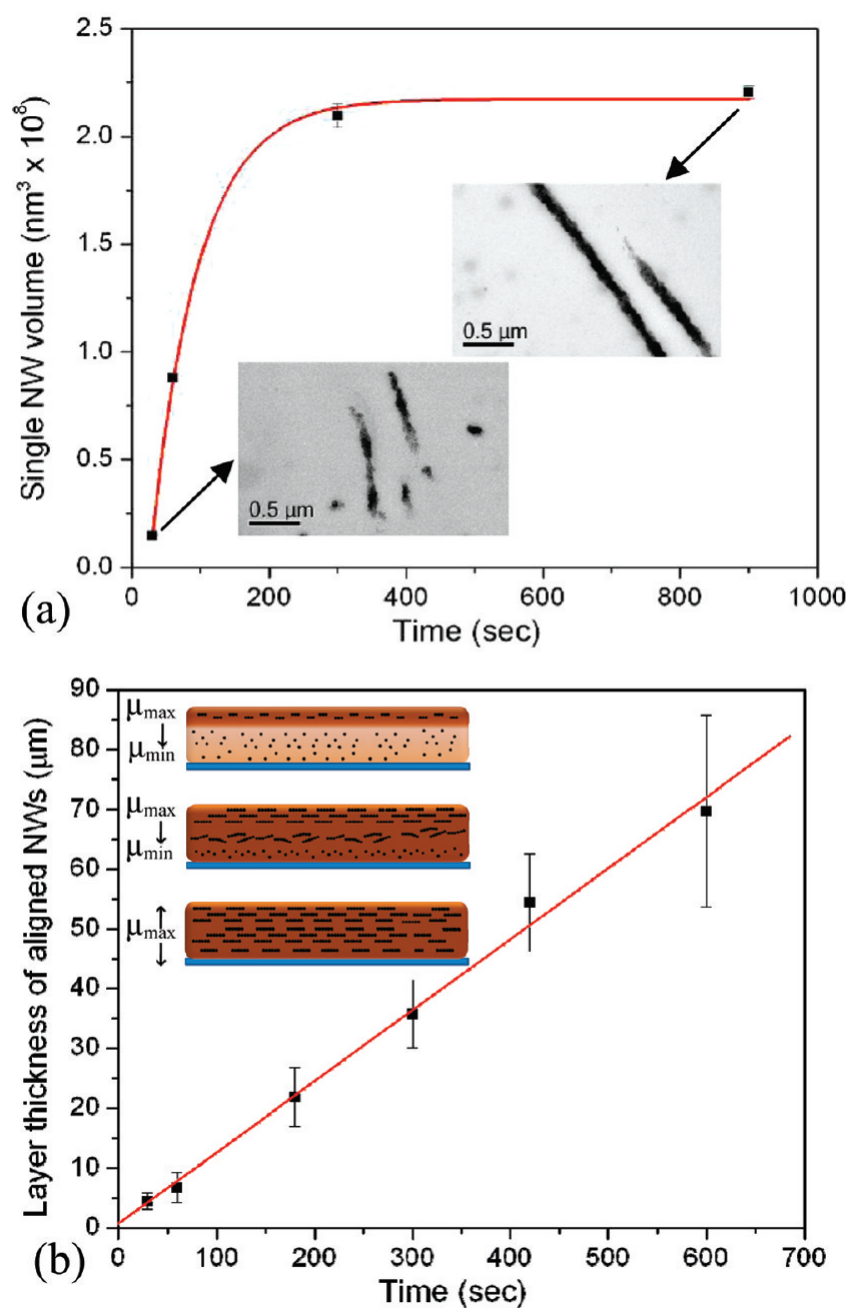

Figure 3. (a) Change of the mean NW volume, as a function of the MF application time; (inset) BF-TEM images of the NWs formed after $30 \mathrm{~s}$ of MF time application (bottom), and after $15 \mathrm{~min}$ (top). (b) Evolution of the thickness of film layer containing aligned NWs (measured from the top surface using an optical microscope) as a function of MF application time; (inset) scheme of the change in viscosity and of the formed chain structures throughout the various layers. aggregation ${ }^{19}$ as required in the initial stage of NW formation. (Figure 2a,b)

To follow the evolution of the formation of NW arrays at different depths in the polymer film, increasing time intervals of MF application (from $30 \mathrm{~s}$ to $15 \mathrm{~min}$ ) after the deposition of the solutions onto the glass substrate have been considered. For short times ( $<60 \mathrm{~s}$ ), the NPs form elongated structures (up to 700 $\mathrm{nm}$ ) aligned parallel to the magnetic field lines. Prolongation of the MF application time results in NWs with increasing length (from $\sim 700 \mathrm{~nm}$ to $\sim 15 \mu \mathrm{m}$ ), while their diameter remains constantly around $80 \mathrm{~nm}$ since the beginning of the process (Figure 3a). Thus, it is clear that under MF application any tendency of the initially formed NP-clusters to aggregate in a random way due to complicated angular dependence of dipolar forces is prevented, whereas head-to-tail chain formation along the magnetic field lines prevails. ${ }^{20}$ As graphically shown in Figure 3b, the NWs initially are formed at the air-liquid interface, and successively deeper in the film, progressively ordering the aggregated structures across the polymer thickness. Eventually, after $\sim 13 \mathrm{~min}$, all the film volume homogeneously consists of long, well ordered NWs. According to the literature after a polymer solution is casted on a substrate, the polymer concentration is higher close to the liquid-air interface than in the inner layers, due to the faster solvent evaporation..$^{21,22}$ In a simplified model, it can be assumed that mass transfer due to evaporation is responsible for such concentration gradients and consequently viscosity gradients. ${ }^{22}$ Accordingly, the viscosity varies throughout the casted solution during evapo-

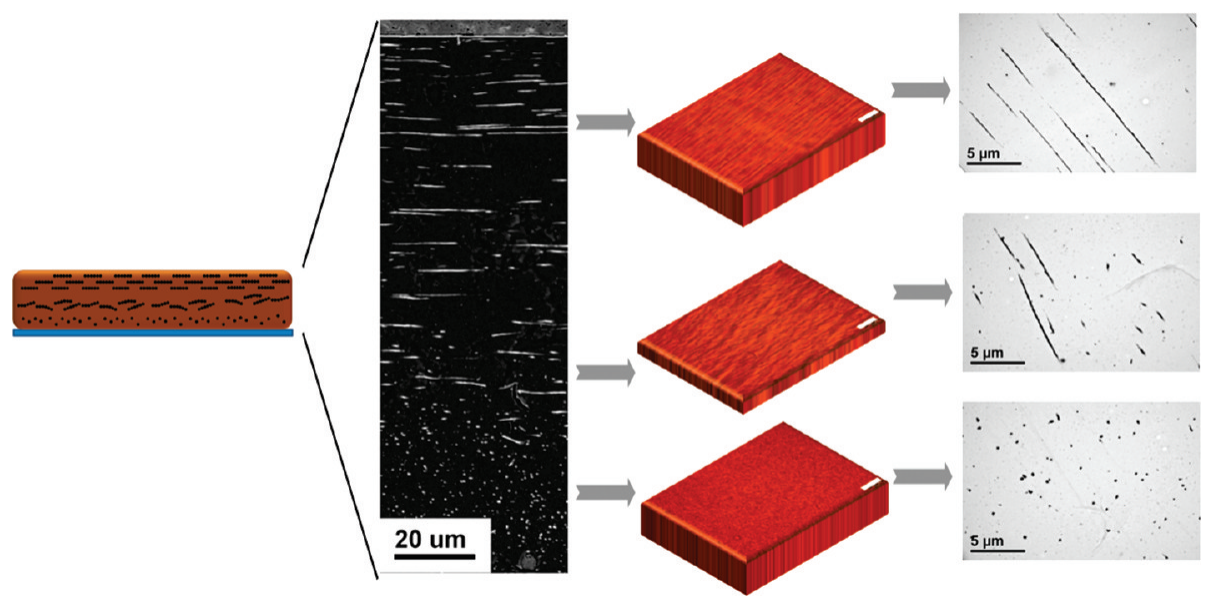

Figure 4. (Left) Scheme and BSE-SEM cross sectional image of the film. The bright lines represent the NWs and the NPs while the dark area is the polymer matrix. (Center) Optical microscope images at various film depths (scale bar $=20 \mu \mathrm{m}$ ). (Right) BF-TEM cross sectional images, demonstrating the NWs at the corresponding depths. (TEM images are rotated about $45^{\circ}$ clockwise with respect to the SEM image). 

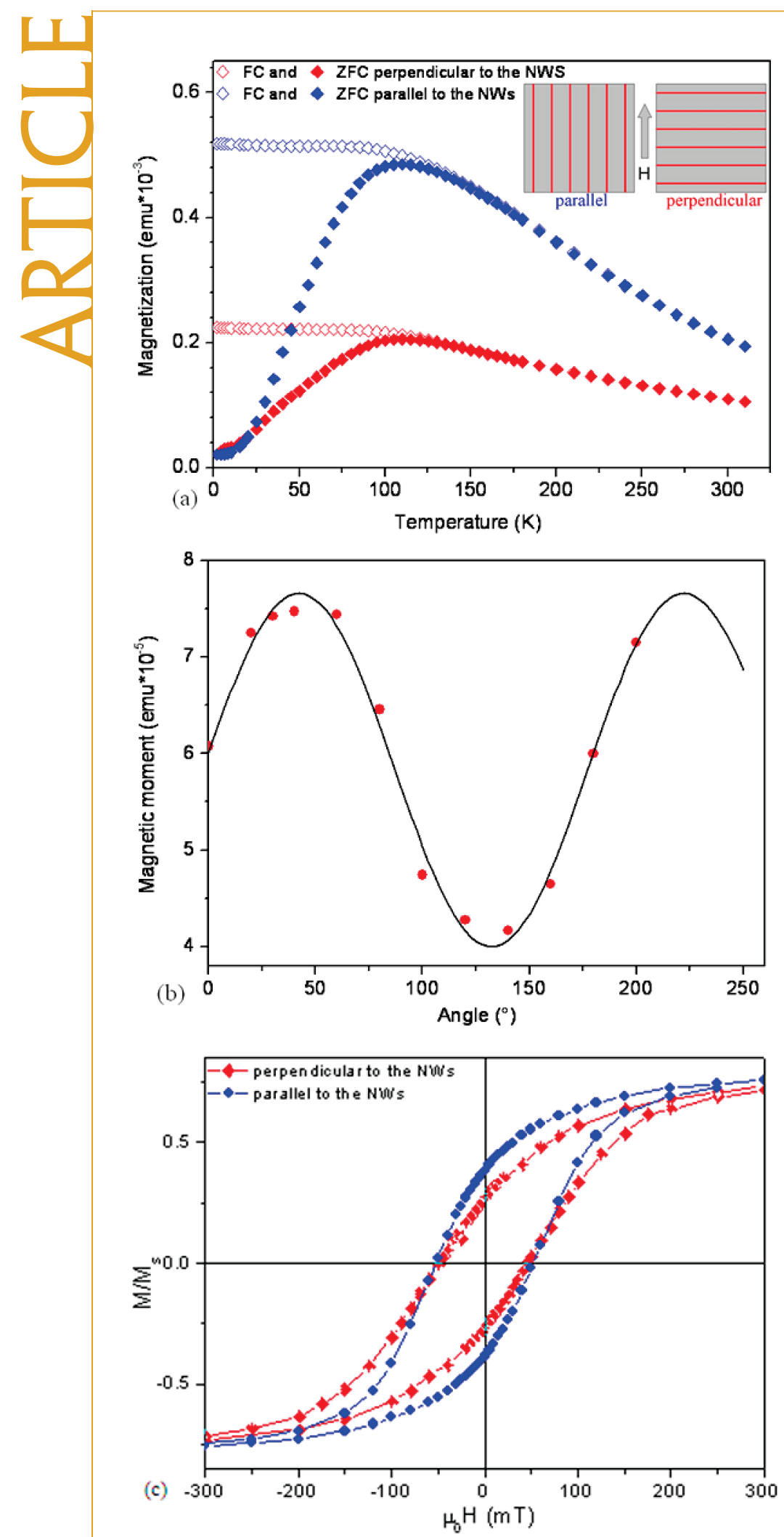

Figure 5. (a) ZFC and FC magnetization curves measured with the direction of the external MF parallel and perpendicular to the aligned NWs as indicated in the inset. (b) Angular dependence of the magnetic moment measured at $5 \mathrm{mT}$ and $300 \mathrm{~K}$. The solid line represents a fit to the law $y=a \sin ^{2}(x$ $+\phi)+b \cos ^{2}(x+\phi)$ with $a=3.99, b=7.67, \phi=-42.1$. (c) Low temperature $(2.5 \mathrm{~K})$ hysteresis loops at both orientations. Magnetization values were normalized to the corresponding saturation values.

ration, being higher close to the surface and decreasing in the inner layers of the film, reaching a minimum

value just above the substrate. We assume a viscosity value $\left(\mu_{\max }\right)$, above which NW formation becomes irreversible due to reduced mobility. An increase in the evaporation time causes the increase of the thickness of the layer with viscosity $\mu_{\max }$. Figure $3 \mathrm{~b}$ illustrates that the layer thickness of the dry film (where viscosity has reached the $\mu_{\max }$ ) where the NWs are observed increases linearly with the MF application time in the first minutes.

More specifically as shown in Figure 3, after $30 \mathrm{~s}$ in the casted solution elongated clusters $\sim 700 \mathrm{~nm}$ long are formed, partially aligned along the field direction. During these first seconds only a thin layer close to the surface has reached the viscosity $\mu_{\max }$ forcing the elongated structures to remain aligned even after the removal of the field. In the rest of the casted solution, where the viscosity is lower, after the MF removal the enhanced Brownian motion causes the elongated NP-chain to break into the constituent parts. ${ }^{23}$ As the MF application time increases, NWs are formed that remain aligned after the MF removal throughout the film volume where $\mu \geq \mu_{\max }$ (after $1 \mathrm{~min}$ the NW average length is $\sim 4$ $\mu \mathrm{m}$, and above $5 \mathrm{~min}$ it is $\sim 15 \mu \mathrm{m}$ ). In the remainder of the casted solution as the gradient of the viscosity decreases, reaching the minimum value $\mu_{\text {min }}$ just above the substrate, the NWs break into elongated chains with reduced length and degree of orientation as they approach the substrate after the MF removal (Figure 4). The thickness of the layer with $\mu \geq \mu_{\max }$ increases with MF application time up to $\sim 13 \mathrm{~min}$ where all the film consists of long well-aligned NWs. In this case, after the removal of the field, the viscosity is high enough everywhere and the Brownian motions are too weak to cause any deformation.

A magnetic study of the films demonstrates that the NWs embedded in the polymer are magnetically anisotropic, while maintaining room temperature superparamagnetic behavior, like that of the pristine $\gamma-\mathrm{Fe}_{2} \mathrm{O}_{3}$ nanocrystals (see Supporting Information Figures S5, S6). This is well demonstrated by the thermal reversibility observed above ca. $140 \mathrm{~K}$ in the magnetization recorded as a function of temperature after zero-field-cooled (ZFC) and field-cooled (FC) procedures (Figure 5a). Moreover the magnetizations of the structured films after application of a static MF parallel and perpendicular to the NW alignment direction shows a strong directional dependence with the temperature. The magnetization is clearly higher in the parallel orientation, which is consistent with the different roles of internal fields expected for the two orientations ${ }^{10}$ (Figure 5a). The anisotropy is better evidenced by measuring the angular dependence of the magnetic moment, which shows an increase of $c a$. $90 \%$ upon rotating the film 
from the perpendicular to the parallel orientation (Figure 5b). Additionally, the hysteresis loops measured in the blocked state $(2.5 \mathrm{~K})$ show that the coercive field and the normalized remanence are higher in the parallel than in the perpendicular direction (Figure $5 \mathrm{c}$ ), which can be due to both dipolar interactions and to a partial alignment of the magnetic NP easy axis along this direction. ${ }^{7,10,24}$ Moreover the hysteresis loop recorded with the MF perpendicular to the NWs both in the plane and out of the plane of the film are identical (see Supporting Information Figure S7), indicating that magnetic interactions among NWs are negligible. The comparison with magnetization studies from other groups on NWs made by magnetic assembly of maghemite NPs not embedded in a polymer matrix $^{7}$ showed that the magnetization properties in both directions are similar to the ones reported here, indicating that the magnetic properties of the derived nanocomposite films are weakly affected by the polymer matrix.
In conclusion, we have presented the formation of magnetic NWs in polymer matrices, with controlled dimensions, localized at specific layers in the polymer matrix, by varying the MF application time. The polymer matrix plays an important role since it induces the aggregation process in the initial stage of formation and defines a viscosity gradient in the film volume, contributing to the irreversible formation of welldetermined NWs at specific depths in the film. The obtained films can be freestanding, and show magnetic anisotropy, with higher magnetization in the direction of the NWs growth. The method may be expanded to other polymer-NPs systems, depending on the desired application, and the resulting films may be used as models for the theoretical exploration of the forces and interactions taking place in the formation procedure. Moreover, the possibility of having NWs of different lengths aligned in various layers implies different magnetic behavior in the same film opening the way for the application of this advanced multilayer structure in magnetic devices. ${ }^{25}$

\section{METHODS}

NPs Synthesis. Nearly spherical mixed-phase $\gamma-\mathrm{Fe}_{2} \mathrm{O}_{3} / \mathrm{Fe}_{3} \mathrm{O}_{4}$ NPs of $10 \mathrm{~nm}$ diameter (see Figure S1 in Supporting Information) were synthesized by modifying a wet-chemical synthetic approach to shape-controlled spinel-cubic iron oxide NPs reported in the literature. ${ }^{26}$ Briefly, iron pentacarbonyl $\left(\mathrm{Fe}(\mathrm{CO})_{5}, \mathrm{Al}\right.$ drich $97 \%$ ) was used as the precursor and decomposed in 1-octadecene (ODE, Aldrich $90 \%$ ) at $280^{\circ} \mathrm{C}$ under inert atmosphere in the presence of oleic acid (OLAC, Aldrich 90\%), oleyl amine (OLAM, Aldrich 97\%) and hexadecane 1,2-diol (HDIOL, Aldrich $98 \%$ ) as both capping molecules and reactivity moderators. ${ }^{26}$ In a typical preparation of 7-nm NPs, $30 \mathrm{~g}$ of ODE, $3 \mathrm{mmol}$ of HDIOL, $1.8 \mathrm{mmol}$ of OLAM, $1.8 \mathrm{mmol}$ of OLAC, and $4 \mathrm{mmol}$ of $\mathrm{Fe}(\mathrm{CO})_{5}$ were comixed and heated up at $280^{\circ} \mathrm{C}$ for $2 \mathrm{~h}$ under $\mathrm{N}_{2}{ }^{26}$ To grow larger NPs with a diameter of $10 \mathrm{~nm}$, a seeding approach was used. ${ }^{27}$ The crude reaction mixture containing 7-nm NPs was cooled to $120^{\circ} \mathrm{C}$ and an extra $4 \mathrm{mmol}$ of $\mathrm{Fe}(\mathrm{CO})_{5}$ was added to it, followed by further heating at $280^{\circ} \mathrm{C}$ for $1 \mathrm{~h}$ under $\mathrm{N}_{2}$ and at $80^{\circ} \mathrm{C}$ for $1 \mathrm{~h}$ under air. After extraction/purification procedures, the hydrophobic NPs resulted in being soluble in nonpolar media, such as chloroform, toluene, and hexane. According to Fourier transform infrared spectroscopy (FTIR) measurements, the NPs mainly retain an oleate ligand capping bound to their surface, although the presence of OLAM and HDIOL cannot be excluded (Figure S8 in Supporting Information). This is in agreement with previous FTIR studies on iron oxide NPs synthesized by similar high-temperature surfactant-assisted nonhydrolytic routes. ${ }^{27,28}$

Films Preparation. $1 \mathrm{mM}$ solution of an acrylate copolymer (poly(ethylmethacrylate-co-methylacrylate), PEMMA) in chloroform was prepared by dissolving PEMMA at a concentration of $100 \mathrm{mg} / \mathrm{mL}$. For the results presented in the manuscript, mixtures containing 1 wt \% of presynthesized NPs and 99 wt \% of PEMMA were used. After being sonicated for $10 \mathrm{~min}$ to avoid the formation of aggregates, the resulting solution was drop casted on a glass substrate. As needed, the system was subjected to a homogeneous MF ( $\sim 160 \mathrm{mT})$, produced by two permanent magnets, applied parallel to the substrate during the deposition and evaporation process. For the kinetic study the films were exposed to the MF for different time intervals upon deposition of the solution on the substrates. After the removal of the MF all films were allowed to dry overnight. The magnetic mea- surements were performed on films in which NWs were present in the whole volume.

Samples Characterization. The films were studied at low magnification with an optical microscope (Olympus BX41). Higher magnification investigations were performed via scanning electron microscopy (SEM) with a JEOL JSM-6490LA microscope detecting the backscattered electrons (BSE) and via transmission electron microscopy (TEM) with JEOL JEM-1011 microscope operating at $100 \mathrm{kV}$ in bright-field (BF) mode. The investigated samples were thin film sections with thicknesses about 500 and $120 \mathrm{~nm}$, respectively, which were cut with a Leica EM UC6 Ultramicrotome. The NWs average length and diameter were measured from SEM and TEM images, averaging manually at least 30 wires. Automated analysis with image processing software (ImageJ) ${ }^{29}$ gave slightly higher values for the diameters, probably systematically affected by residual particles from the cutting procedure surrounding the NWs. The percentage of the NWs into the volume of the film was considered by using the TEM and SEM images of thin film slices $(500 \mathrm{~nm})$ and calculating the ratio of the volume of NWs to the volume of the slice. The magnetic properties were studied with a Quantum Design Ltd. SQUID magnetometer. The temperature dependence of ZFC-FC magnetization was collected in a static field of $10 \mathrm{mT}$ after cooling the samples down to $2 \mathrm{~K}$ in a zero magnetic field (ZFC curve) or in the same probe field of $10 \mathrm{mT}$ (FC curve). The angular dependence of the magnetization was measured at $300 \mathrm{~K}$ by rotating the sample by $20^{\circ}$ steps, while keeping the $5 \mathrm{mT}$ applied field in the plane of the film. The hysteresis curves were measured at $2.5 \mathrm{~K}$ with applied fields in between $\pm 5 \mathrm{~T}$. All the measurements were performed applying the field in the plane of the slab, first parallel and then perpendicular to the direction of the aligned NWs in the polymer matrix. All data were corrected for the diamagnetic contributions of the polymer and of the substrate, which were separately measured and which were found negligible.

Supporting Information Available: Figure S1: Low-resolution TEM image of the as-synthesized $\gamma-\mathrm{Fe}_{2} \mathrm{O}_{3}$ magnetic NPs. Figure S2: Optical microscope images of various colloidal NPs/polymer assemblies. Figure S3: Optical microscope images of $\gamma-\mathrm{Fe}_{2} \mathrm{O}_{3} \mathrm{NP}$ / PEMMA assemblies with different NP concentrations. Figure S4: Optical image of $\gamma-\mathrm{Fe}_{2} \mathrm{O}_{3}$ NP assembly on glass substrate in the absence of polymer. Figure S5: Hysteresis loops at $2.5 \mathrm{~K}$ of a 
$\gamma-\mathrm{Fe}_{2} \mathrm{O}_{3} \mathrm{NP}$ solution in chloroform. Figure S6: ZFC-FC magnetizations of the $\gamma-\mathrm{Fe}_{2} \mathrm{O}_{3}$ frozen solution. Figure $\mathrm{S} 7$ : Hysteresis loops for 1 wt \%. $\gamma-\mathrm{Fe}_{2} \mathrm{O}_{3} /$ PEMMA film. Figure S8: FTIR spectra of $\gamma-\mathrm{Fe}_{2} \mathrm{O}_{3} \mathrm{NPs}$. This material is available free of charge via the Internet at http://pubs.acs.org.

\section{REFERENCES AND NOTES}

1. Weller, D.; Doerner, M. F. Extremely High-Density Longitudinal Magnetic Recording Media. Annu. Rev. Mater. Sci. 2000, 30, 611-644.

2. Kaushik, A.; Solanki, P. R.; Ansaria, A. A.; Sumanaa, G.; Ahmadb, S.; Malhotraa, B. D. Iron Oxide-Chitosan Nanobiocomposite for Urea Sensor. Sens. Actuators $B$ 2009, 138, 572-580

3. Fahrni, F.; Prins, M. W. J.; van ljzendoorn, L. J. Magnetization and Actuation of Polymeric Microstructures with Magnetic Nanoparticles for Application in Microfluidics. J. Magn. Magn. Mater. 2009, 321, 1843-1850.

4. Merkel, T. C.; Freeman, B. D.; Spontak, R. J.; He, Z.; Pinnau, I.; Meakin, P.; Hill, A. J. Ultrapermeable, Reverse-Selective Nanocomposite Membranes. Science 2002, 296, 519-522.

5. Huo, J.; Wang, L.; Yu, H. Polymeric Nanocomposites for Electromagnetic Wave Absorption. J. Mater. Sci. 2009, 44, 3917-3927.

6. Tang, Z.; Kotov, N. A. One-Dimensional Assembly of Nanopartilcles: Preparation, Properties and Promise. Adv. Mater. 2005, 17, 951-962.

7. Lalatonne, Y.; Motte, L.; Russier, V.; Ngo, A. T.; Bonville, P.; Pileni, M. P. Mesoscopic Structures of Nanocrystals: Collective Magnetic Properties Due to the Alignment of Nanocrystals. J. Phys. Chem. B 2004, 108, 1848-1854.

8. Park, J. I.; Jun, Y. W.; Choi, J. S.; Cheon, J. Highly Crystalline Anisotropic Superstructures via Magnetic Field Induced Nanoparticle Assembly. Chem. Commun. 2007, 5001-5003.

9. Fang, W. X.; He, Z. H.; Xu, X. Q.; Mao, Z. Q.; Shen, H. Magnetic-Field-Induced Chain-like Assembly Structures of $\mathrm{Fe}_{3} \mathrm{O}_{4}$ Nanoparticles. Europhys. Lett. 2007, 77, 68004.

10. Sahoo, Y.; Cheon, M.; Wang, S.; Luo, H.; Furlani, E. P.; Prasad, P. N. Field-Directed Self-Assembly of Magnetic Nanoparticles. J. Phys. Chem. B 2004, 108, 3380-3383.

11. Pileni, M. P. Magnetic Fluids: Fabrication, Magnetic Properties, and Organization of Nanocrystals. Adv. Funct. Mater. 2001, 11, 323-336.

12. Goubault, C.; Leal-Calderon, F.; Viovy, J.-L.; Bibette, J. SelfAssembled Magnetic Nanowires Made Irreversible by Polymer Bridging. Langmuir 2005, 21, 3725-3729.

13. Geng, B. Y. Hydrophilic Polymer Assisted Synthesis of Room-Temperature Ferromagnetic $\mathrm{Fe}_{3} \mathrm{O}_{4}$ Nanochains. Appl. Phys. Lett. 2007, 90, 043120.

14. Evans, B. A.; Shields, A. R.; Lloyd Carroll, R.; Washburn, S.; Falvo, M. R.; Superfine, R. Magnetically Actuated Nanorod Arrays as Biomimetic Cilia. Nano Lett. 2007, 7, 1428-1434.

15. Martin, J.; Mijangos, C. Tailored Polymer-Based Nanofibers and Nanotubes by Means of Different Infiltration Methods into Alumina Nanopores. Langmuir 2009, 25, 1181-1187.

16. Martin, J. E.; Venturini, E.; Odinek, J.; Anderson, R. A. Anisotropic Magnetism in Field-Structured Composites. Phys. Rev. E 2000, 61, 2818-2830.

17. Jestin, J.; Cousin, F.; Dubois, l.; Ménager, C.; Schweins, R.; Oberdisse, J.; Boué, F. Anisotropic Reinforcement of Nanocomposites Tuned by Magnetic Orientation of the Filler Network. Adv. Mater. 2008, 20, 2533-2540.

18. Lalatonne, Y.; Richardi, J.; Pileni, M. P. Van der Waals versus Dipolar Forces Controlling Mesoscopic Organizations of Magnetic Nanocrystals. Nat. Mater. 2004, 3, 121-125.

19. Sciancalepore, C.; Cassano, T.; Curri, M. L.; Mecerreyes, D.; Valentini, A.; Agostiano, A.; Tommasi, R.; Striccoli, M. TiO Nanorods/PMMA Copolymer-Based Nanocomposites: Highly Homogeneous Linear and Nonlinear Optical Material. Nanotechnology 2008, 19, 205705.

20. Tanase, M.; Bauer, L. A.; Hultgren, A.; Silevitch, D. M.; Sun L.; Reich, D. H.; Searson, P. C.; Meyer, G. J. Magnetic Alignment of Fluorescent Nanowires. Nano Lett. 2001, 1, 155-158.
21. Tsige, M.; Grest, G. S. Solvent Evaporation and Interdiffusion in Polymer Films. J. Phys.: Condens. Matter 2005, 17, S4119-S4132.

22. Jenekhe, S. A. Effects of Solvent Mass Transfer on Flow of Polymer Solutions on a Flat Rotating Disk. Ind. Eng. Chem. Fundam. 1984, 23, 425-432.

23. Furst, E. M.; Suzuki, C.; Fermigier, M.; Gast, A. P. Permanently Linked Monodisperse Paramagnetic Chains. Langmuir 1998, 14, 7334-7336.

24. Alphandry, E.; Ding, Y.; Ngo, A. T.; Wang, Z. L.; Wu, L. F.; Pileni, M. P. Assemblies of Aligned Magnetotactic Bacteria and Extracted Magnetosomes: What Is the Main Factor Responsible for the Magnetic Anisotropy. ACS Nano 2009, 3, 1539-1547.

25. MacKenzie, M.; Chapman, J. N.; Cardoso, S.; Li, H.; Ferreira, R.; Freitas, P. P. Analytical Electron Microscopy of Advanced Multilayer Structures for Magnetic Devices. J. Phys. D: Appl. Phys. 2005, 38, 1869-1874.

26. Cozzoli, P. D.; Snoeck, E.; Garcia, M. A.; Giannini, C.; Guagliardi, A.; Cervellino, A.; Gozzo, F.; Hernando, A.; Achterhold, K.; Ciobanu, N.;. et al. Colloidal Synthesis and Characterization of Tetrapod-Shaped Magnetic Nanocrystals. Nano Lett. 2006, 6, 1966-1972.

27. Sun, S.; Zeng, H.; Robinson, D. B.; Raoux, S.; Rice, P. M.; Wang, S. X.; Li, G. Monodisperse $\mathrm{MFe}_{2} \mathrm{O}_{4}(\mathrm{M}=\mathrm{Fe}, \mathrm{Co}, \mathrm{Mn})$ Nanoparticles. J. Am. Chem. Soc. 2004, 126, 273-279.

28. Willis, A. L.; Turro, N. J.; O'Brien, S. Spectroscopic Characterization of the Surface of Iron Oxide Nanocrystals. Chem. Mater. 2005, 17, 5970-5975.

29. Rasband, W. S. ImageJ; U.S. National Institutes of Health: Bethesda, Maryland, 1997-2009; http://rsb.info.nih.gov/ij/. Accessed February 18, 2010. 